\title{
La generación interactiva en Colombia: adolescentes frente a la Internet, el celular y los videojuegos ${ }^{*}$
}

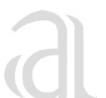 \\ Germán Arango Forero** \\ Xavier Bringué Sala \\ Charo Sádaba Chalezquer
}

Recibido: 23 de agosto de 2010 Aprobado: 25 de septiembre de 2010

\begin{abstract}
Resumen
La penetración de nuevas tecnologías de información y comunicación, tales como Internet, telefonía celular y videoconsolas, ha experimentado un crecimiento sostenido en Colombia durante la primera década del nuevo siglo. Los menores de edad, y entre ellos los adolescentes, integran el grupo poblacional más dispuesto para adoptar y adaptar, con mayor naturalidad, estas nuevas herramientas comunicativas en su vida diaria. El estudio sobre sus hábitos de consumo y sus preferencias en el uso de este tipo de nuevos medios de comunicación es el objetivo central del presente artículo de investigación.
\end{abstract}

Palabras clave: Colombia, Internet, celular, videoconsolas, adolescentes, acceso, uso, consumo

Este artículo de investigación científica y tecnológica hace parte de la investigación La generación interactiva en Colombia: Adolescentes frente a Internet, el celular y los videojuegos, patrocinada por el Foro Generaciones interactivas.

Doctorando en Ciencias de la Comunicación. Perfeccionamiento Institute for Media and Entertainment Management. Comunicador social-periodista. Universidad de la Sabana, Bogotá, Colombia.

... Licenciado en Ciencias de la Educación y doctor en Comunicación. Universidad de Navarra, España.

"... Doctora en Comunicación. Subdirectora del Laboratorio de Comunicación Multimedia. Profesora Adjunta de las asignaturas de Proyectos Publicitarios, Nuevos Medios Publicitarios y Marketing Especializado. Universidad de Navarra, España. 


\title{
Interactive generation in Colombia: teenagers before the internet, the cell phone, and the videogames
}

\begin{abstract}
Information and Communication Technologies (ICT'S) such as Internet, Cell Phones and Videoconsoles have risen stably in Colombia during the first decade of the 21 st Century. Kids, adolescents and young people tend to adapt and use these new communicative platforms more naturally in their daily lives. The study on their consumption and uses habits of the new ICT's technologies is the main goal of this research article.
\end{abstract}

Key words: Colombia, Internet, celular, videoconsoles, adolescents, access, new media, uses, consumption 


\section{Introducción}

El final de la primera década del nuevo siglo situó a Colombia con el mayor crecimiento de América Latina en cuanto a penetración de Internet. A finales de 2009, el país registró un incremento del $32 \%$ frente al 23\% consolidado de la región (Fosk, 2010). Una realidad muy diferente a la registrada durante los primeros años de la nueva década, en los que el país se situó a la saga en cuanto a la implementación de nuevas tecnologías de comunicación. Pero una política oficial de apoyo al sector, sumada a un clima favorable para la inversión extranjera atraída por un mercado potencial muy grande, fueron factores que le permitieron avanzar al país en materia de telecomunicaciones en los principales centros urbanos. No obstante, los retos siguen siendo grandes en materia de cobertura de Internet de banda ancha, especialmente entre los grupos socioeconómicos bajos, en municipios pequeños y en las zonas rurales (Arango Forero, 2008).

En el ámbito regional Latinoamérica también experimentó durante la primera década del siglo XXI un crecimiento sostenido en telefonía celular. A 2008 la base de suscriptores superaba los 300 millones en tecnología GSM y 69,2 millones en plataforma CDMA, con proyecciones de crecimiento por encima de los 500 millones de suscriptores para el año 2012, con un promedio superior a las 80 líneas por cada 100 habitantes; Argentina, Uruguay, Venezuela, Chile, Ecuador y Colombia son los países con el registro de mayor cobertura en la región (Ontiveros Baeza, Martín Enríquez, Fernández de Lis, Rodríguez Téubal, \& López Sabater, 2009)

Todos estos números, susceptibles de perder vigencia rápidamente ante la dinámica creciente del fenómeno conocido como tecnologías de información y comunicación (TIC), indican la presencia de un nuevo panorama de compleja oferta comunicativa, con su consecuente efecto en la tradición del consumo.

En el campo de la industria mediática audiovisual, la proliferación de las consideradas "nuevas pantallas" sitúa a los niños, adolescentes y jóvenes como los naturales adoptantes de estas nuevas tecnologías, consideradas incluso por estos segmentos poblacionales como algo natural, en su consumo mediático cotidiano.

La denominada generación interactiva se destaca, entre otras cosas, por su evidente afinidad con Internet y los nuevos medios. Dicha afinidad se debe a que las Tecnologías de la Información y la Comunicación encuentran en niños y adolescentes un terreno especialmente abonado para su rápida implantación (Bringué, 2008, p.25).

Vivimos en un mundo en el que niños, adolescentes y jóvenes mantienen relaciones de todo tipo a través de las pantallas. Esta nueva situación hace que surjan nuevos retos desde el punto de vista de la investigación. Uno de los principales desafíos en este campo de estudio quizá sea analizar las pautas de consumo, uso y valoración propias de esta peculiar audiencia en lo relativo a las pantallas señaladas.

Este artículo se encuadra dentro de este marco de análisis. La investigación recogida en él pretende dar respuesta a estas preguntas, centrando su caso de estudio en la realidad experimentada al respecto por los menores colombianos. El estudio realizado describe el escenario en el que actúa la generación interactiva colombiana y analiza la relación que mantienen con Internet, los celulares y los videojuegos los adolescentes con edades comprendidas entre los 10 y 18 años, quienes habitan en los principales centros urbanos del país.

Aunque la televisión (tanto abierta como de pago) sigue siendo el medio dominante y predominante para el consumo audiovisual entre adolescentes y jóvenes colombianos (Arango Forero E González Bernal, 2009), resulta igualmente evidente la presencia de las nuevas pantallas en el consumo mediático entre estos segmentos de edad.

\section{Metodología}

El presente estudio se extrae de una investigación realizada en siete países de América Latina: Argentina, Brasil, Colombia, Chile, México, Perú, y Venezuela. El universo de estudio está formado por la población escolarizada adolescente y la muestra total investigada ha sido de 25.467 sujetos. En el 
caso de Colombia, la muestra incluye a un total de 3.292 escolares, entre 10 y 18 años, residentes en ámbitos urbanos y estudiantes de colegio.

Se realizó un muestreo multietápico que combina muestreo estratificado y por conglomerados. En primer lugar, se eligieron al azar las zonas urbanas del país, agrupadas según la población escolarizada, tanto privada como pública. El margen de error muestral es del 2,5\%.

La técnica utilizada para llevar a cabo la presente investigación es la encuesta online a través de un cuestionario autoadministrado, compuesto por un total de 60 preguntas y alojado en la página web www.generaciones-i.net/

La recogida de datos a través del cuestionario online se realizó a través de la red: los escolares participantes respondieron a las diversas preguntas desde las aulas de informática de su propio centro educativo. A cada centro se le asignó un código de participación para controlar el acceso de los encuestados y, al mismo tiempo, proteger su identidad personal. Además, para asegurar la calidad de la información recopilada se limitó el tiempo de respuesta, contando con un profesor en el aula. También se midió la repetición de las respuestas para evitar la contabilización de participaciones múltiples por parte de un mismo usuario.

\section{Acceso y uso de Internet}

$48,6 \%$ de los adolescentes colombianos manifestaron no tener acceso a Internet desde el hogar, frente a un 36\% que aseguró disponer de conexión desde su vivienda, porcentaje casi 10 puntos inferior a la media latinoamericana de conexión, situada en el 45,9\%. El acceso a Internet desde casa es considerablemente superior entre las mujeres, con un $42,4 \%$, frente a un $27,5 \%$ de los hombres.

A pesar de que las tasas de acceso a Internet desde el hogar no son elevadas en Colombia, es relevante destacar que no poseer conexión propia no implica la ausencia del uso de la red. Como demuestran los datos obtenidos, es frecuente que los adolescentes se conecten a la red desde otros lugares, incluso teniendo acceso desde el hogar. Ante la pregunta de "¿En qué lugar sueles usar Internet?", la mayoría afirmó hacerlo desde el colegio, 45,9\%. El segundo lugar más frecuente de conexión es el llamado Café Internet, o Cibercafé, con el 39,8\%, seguido a distancia considerable por el hogar con 30,3\%. Otros sitios de conexión también fueron referidos, como lo demuestra la siguiente tabla.

Tabla 1. Lugares de acceso a Internet

\begin{tabular}{|l|r|r|r|r|r|r|}
\hline & \multicolumn{1}{|c|}{$\begin{array}{c}\text { Casa } \\
(\%)\end{array}$} & $\begin{array}{c}\text { Colegio } \\
(\%)\end{array}$ & $\begin{array}{c}\text { Ciber } \\
(\%)\end{array}$ & $\begin{array}{c}\text { Casa } \\
\text { amigo } \\
(\%)\end{array}$ & $\begin{array}{c}\text { Casa } \\
\text { familiar } \\
(\%)\end{array}$ & $\begin{array}{c}\text { Otro } \\
\text { sitio } \\
(\%)\end{array}$ \\
\hline Masculino & 23.3 & 44,6 & 43,20 & 22,6 & 19,1 & 16,3 \\
\hline Femenino & 36 & 46,9 & 37,4 & 28 & 25,3 & 16,2 \\
\hline Total & 30,2 & 45,9 & 39,8 & 25,5 & 22,6 & 16,4 \\
\hline
\end{tabular}

Fuente: Encuesta Generaciones Interactivas en Iberoamérica. Respuesta a la pregunta № 14 "¿En qué lugar sueles usar Internet?". $\mathrm{N}=3.292$ escolares de 10 a 18 años.

El porcentaje femenino que se conecta desde el hogar supera al de los varones en más de 13 puntos, mientras que ellos destacan frente a las mujeres en las conexiones desde cibercafés. También se destaca que el porcentaje de mujeres que se conectan a la red desde la casa de un amigo, o desde la casa de un familiar, es superior en ambos casos al de los hombres. El colegio y 'otro sitio' son, por el contrario, los dos lugares donde hay una mayor coincidencia porcentual entre ambos géneros.

Los adolescentes forman parte de la denominada generación interactiva y han nacido rodeados de toda suerte de posibilidades tecnológicas antes no conocidas. Esto supone que, para ellos, todo aquello que los adultos denominamos nuevos medios no lo sean en absoluto. Para un adolescente, Internet es un medio convencional. Por consiguiente, tiene sentido pensar que, en numerosos casos, Internet convive con variadas actividades realizadas por los adolescentes; en unos casos se integran en el uso de la Red y en otros son desplazadas.

De hecho, un 29,8\% de la muestra analizada asegura que no le han quitado tiempo a nada para poder utilizar la red. El porcentaje de hombres que se suman a esta afirmación es considerablemente inferior al de las mujeres, 25,3\%, frente al $33,1 \%$. 
Por otra parte, es interesante mencionar que quienes sí consideran que Internet ha supuesto para ellos un desplazamiento de otras actividades, se refieren a que durante ese tiempo podrían estar haciendo otra cosa, pero no necesariamente que hayan dejado de hacerla para volcarse en su computador. En este sentido, podríamos destacar que la principal actividad desplazada por el uso de Internet en Colombia es el estudio, seguida de la televisión y la familia.

Como muestra el gráfico 1 , el género no es un factor que marque diferencias en lo que concierne al desplazamiento de actividades. Las dos únicas excepciones a esta afirmación son los videojuegos y el novio/a. En el caso de los videojuegos, son más del doble los hombres frente a las mujeres los que afirman haberle dado tiempo a esta actividad. Este dato resulta lógico conociendo, como se verá en próximos apartados, el sesgo masculino que tienen los videojuegos en este país. En el caso del tiempo dedicado a la pareja, casi el doble de hombres con relación al de mujeres admite haberle restado tiempo.

Gráfico 1. Desplazamiento de actividades por el uso de Internet

A qué le han quitado tiempo (10.18 años)

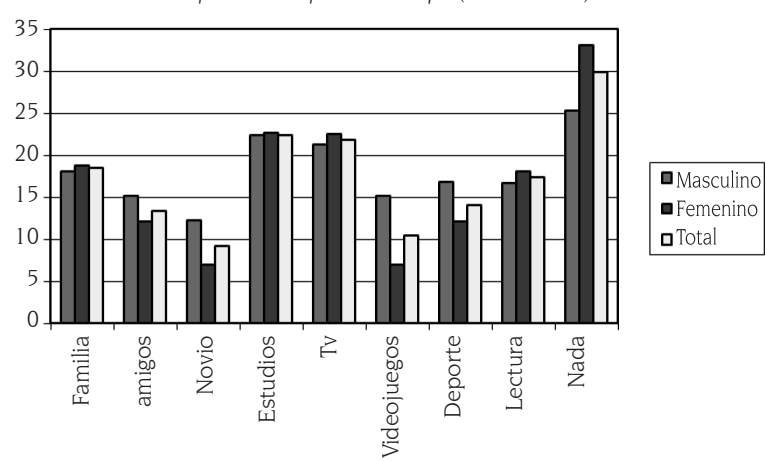

Fuente: Encuesta Generaciones Interactivas en Iberoamérica. Respuestas a la pregunta $\mathrm{N}^{\circ} 24 . \mathrm{N}=3.292$ escolares de 10 a 18 años.

En cuanto al tiempo de conexión, los dos hechos más destacados son la gran cantidad de espacio temporal destinada a esta actividad entre quienes disponen de red en su hogar y el descenso, aunque no muy significativo, del tiempo dedicado a navegar los fines de semana. La gran mayoría de los adolescentes colombianos responden a la tipo heavy users (se conectan más de dos horas diarias). Casi la mitad de la muestra analizada, 45,7\% afirmó conectarse este tiempo a Internet entre semana, porcentaje que decrece sólo hasta el 39\% los fines de semana.

Los medium users (conexión entre una o dos horas diarias) son el segundo grupo más numeroso, tanto entre semana, como los fines de semana. Un 25,4\% de los adolescentes colombianos encuestados admitió destinar este tiempo los días laborables, porcentaje que decrecer poco en fin de semana con el 20,2\%.

Los que se conectan menos de una hora entre semana suponen sólo el 7,8\%, porcentaje que casi se duplica los fines de semana, 14,1\%, rompiendo la tendencia decreciente detectada para los fines de semana entre los heavy y medium users.

En cuanto al género, el porcentaje de mujeres heavy users supera considerablemente al de hombres entre semana $(50,5 \%$ frente al $36 \%$ ); mientras que los hombres consumen más los fines de semana (40,4\% frente al 38,3\%). Justo lo contrario sucede con los mediun users. El porcentaje de hombres que destinan entre una hora y dos horas a navegar entre semana es superior al de mujeres (29,8\% frente al 23,2\%), e inferior los fines de semana (17,8\% frente al $21,3 \%)$.

El $60,4 \%$ de la muestra analizada manifestó que el consumo de Internet se realiza de manera individual. No obstante, en ocasiones también navega en compañía. Un amigo (39,9\%), varios amigos (24\%) y los hermanos (22,5\%) constituyen las compañías principales de los que navegan de este modo, muy por encima de los que se conectan junto a sus padres $(7,5 \%)$, madres $(11,5 \%)$ o profesores $(7,5 \%)$. El porcentaje de mujeres que navegan en presencia de un amigo supera el de hombres en nueve puntos porcentuales: $44,2 \%$ frente al 34,2\%. Los datos aportados en este epígrafe refrendan la certeza de que los adolescentes prefieren relacionarse con aquellos que no suponen para ellos una fuente de autoridad.

\section{Uso de servicios y contenidos}

Los datos obtenidos demuestran que los adolescentes colombianos se caracterizan por utilizar la 
red para multitud de fines. Los más citados en el estudio fueron la posibilidad de comunicarse con otros, el uso con una finalidad informativa y con un carácter lúdico. En cuanto a la finalidad comunicativa, el 56,8\% afirma utilizar el Messenger y más de un 53,3\% el correo electrónico. Un 44,2\% usa la red con una finalidad informativa y la función lúdica también es considerable: el 41,4\% descarga películas y música mientras el 40,1\% juega en red (Gráfico 2, servicios utilizados en Internet).

Gráfico 2. Servicios utilizados en Internet

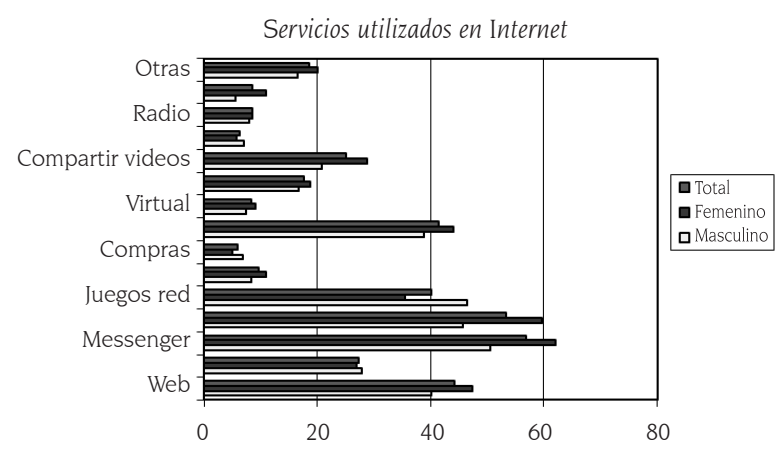

Fuente: Encuestas Generaciones Interactivas en Iberoamérica. Respuestas a la pregunta $N^{\circ} 15$ «ndica cuál de los servicios utilizas cuando navegas por Internet». N=3.292 escolares de 10 a 18 años.

El gráfico demuestra que en muchos de los usos no hay diferencias significativas en cuestión de género. No obstante, a pesar de las semejanzas, se destaca que el porcentaje de mujeres supera siempre al de hombres, especialmente en el empleo del mail y del Messenger, en casi 14 y 12 puntos porcentuales, respectivamente. También merece la pena destacar que casi el doble de mujeres habla por teléfono utilizando la red. Los varones predominan en un porcentaje relevante sobre el uso de los juegos en red: 46,4\% frente al $35,4 \%$.

En cuanto a las páginas visitadas, la música (casi un 68\%), y los juegos a cierta distancia (casi un $50 \%$ ) se erigen como los contenidos más demandados. En cuanto a género, para un 71,4\% de las mujeres la música es el contenido prioritario, frente a un $63,5 \%$ en el caso de los hombres. Por el contrario, el porcentaje masculino es superior en cuanto a las páginas de juegos con un $57,6 \%$ frente, a un $42,6 \%$, respectivamente.

También hay diferencias muy significativas en las visitas de páginas de deportes, 49,7\% de hom- bres, frente al 18,4\% de mujeres; en las páginas para adultos, 6,5\% de hombres, frente al 1,7\% de mujeres; o en las de hobbies o aficiones, donde las féminas aventajan a los varones en más de 7 puntos porcentuales: 19,8\% frente, a 12,2\%. La comparativa por género muestra que las páginas educativas, culturales o de concursos son más visitadas por mujeres que por hombres, aunque los márgenes de distancia entre los géneros son muy inferiores a los destacados en la frase anterior.

\section{Mediación educativa}

Si hablamos de mediación educativa podemos afirmar que Colombia, en este ámbito, está por debajo de las medias latinoamericanas en todas las opciones analizadas. Casi un 34\% de los adolescentes encuestados afirmaron que sus padres 'no hacen nada' mientras ellos navegan, porcentaje dos puntos por debajo de la media obtenida de los resultados del conjunto de los países analizados.

Sin embargo, a pesar de estar por debajo de la media, la mayoría de los adolescentes encuestados evidencia que sí existe algún tipo de mediación, aunque se trate de una simple labor de vigilancia. En este sentido, el 35,8\% de la muestra asegura que sus padres les preguntan qué hacen, mientras que el 26\% afirma que echan un vistazo mientras navegan.

También merece la pena destacar el predominio de la mediación activa (apuntada en el párrafo anterior) frente a la pasiva: sólo un 4,1\% de los adolescentes afirma que sus progenitores comprueban los lugares donde han navegado, mientras que sólo un 3,2\% manifiestan que sus padres miran su correo electrónico.

Por último, consideramos relevante apuntar que el género es un factor que incrementa el porcentaje de mediación existente, aunque la diferencia no es llamativa. Las mujeres que reconocen algún tipo de mediación superan siempre a los hombres en las modalidades de mediación pasiva, aunque la divergencia sólo es considerable en la práctica de 'echar un vistazo': 22,6\% de mujeres, frente al $28,7 \%$ de hombres. En el resto de mo- 
dalidades mediadoras, la distancia entre hombre y mujeres oscila entre las décimas porcentuales y los 4 puntos, siendo siempre superior la mediación pasiva ejercida sobre las mujeres, como ya hemos mencionado. Por el contrario, en modalidades de mediación activa, un 5\% de hombres afirma que sus padres comprueban por dónde han navegado frente a un 3,3\% de mujeres, y un 3,4\% de varones reconoce que sus progenitores revisan su correo electrónico, frente a un 3\% femenino.

Sobre los motivos de discusión, no parece que Internet sea fuente de graves conflictos familiares, más allá del tiempo de conexión, ya que el $44,7 \%$ de la muestra asegura no discutir nunca con sus padres por esta causa. No obstante, sí existe un factor de fricción entre padres e hijos en este sentido: el tiempo que éstos pasan navegando. Un $22,4 \%$ reconocen discutir por este motivo, y es mayor el porcentaje de mujeres que lo hace, $25,3 \%$ frente al $19 \%$ de los hombres.

Y es que los menores parecen tener muy claro qué cosas se les permite hacer mientras navegan y cuáles no. Así, saben que no deben dar información personal, comprar o chatear, tres de las restricciones más reiteradas por parte de los adolescentes colombianos al preguntarles qué les prohíben hacer sus progenitores al conectarse a la Red. Por ello, no es de extrañar que las discusiones no sean demasiado frecuentes. También merece la pena señalar que en lo que a prohibiciones por parte de los padres respecta, los hombres superan a las mujeres en las restricciones referidas a 'jugar', 'subir archivos', 'ver vídeos' y 'enviar correos electrónicos'. En el resto de posibles prohibiciones, el número de mujeres que admiten cada tipo de limitación siempre supera al de hombres. Por ejemplo, si un 32,8\% de los varones admiten la advertencia por parte de sus padres en lo relativo a no facilitar datos personales, el porcentaje se eleva al 43,4\% en el caso de las mujeres.

Con los datos aportados hasta el momento, podemos afirmar que no se puede hablar de tensiones y problemas derivados del uso de Internet por contenidos y prohibiciones quebrantadas, pero probablemente sí en cuanto al tiempo de uso.

\section{Valoraciones}

Sobre la valoración que los adolescentes colombianos tienen de sí mismos como internautas cabría destacar que la mayoría de ellos se reconoce con un nivel medio, 31,1\%. Las mujeres superan a los hombres en todos los niveles, salvo en el de principiantes donde un $24,2 \%$ de los varones se reconoce en ese grado frente al 18,8\% de las féminas.

Un $32,5 \%$ de ellas se sitúa como usuarias de nivel medio; un 24,2\% en el nivel avanzado; y un $9,9 \%$ se autocalifican como expertas. Un $29,7 \%$ de los hombres reconocen tener un nivel medio; un 20,8\% avanzado; y sólo un 7,9\% se consideran expertos. Respaldados por los datos obtenidos en esta investigación parece que en lo que a conocimiento de las TIC respecta, en Colombia, las mujeres aventajan actualmente a los varones.

La investigación realizada también revela que la mayoría de los adolescentes $41,3 \%$, son autodidactos en el uso de Internet. Quienes admiten haber sido instruidos, destacan principalmente a los profesores como mentores en el ámbito, $(27,6 \%)$, siendo algo superior el porcentaje de mujeres, $(29,2 \%)$, que el de hombres $(25,5 \%)$. Este dato refuerza la importancia que los centros educativos tienen en la implementación y la enseñanza de las TIC en Colombia. Ante la evidente ausencia de instituciones orientadoras, gran parte de la formación proviene de personas semejantes. Un 20,5\% de la muestra encuestada afirmó que sus amigos le habían enseñado a usar Internet, mientras que un 17,7\% reconoció que sus hermanos habían sido los instructores en la materia.

\section{Celular}

El teléfono celular es un dispositivo con una penetración muy elevada entre los adolescentes colombianos sin distinción notoria de género. El $88,3 \%$ de los adolescentes encuestados afirma tener este aparato.

El modo más habitual de obtención es a través de los padres, que en su mayoría lo compran ante la insistencia de sus hijos (32,2\%). En otros casos, lo adquieren sin presión o solicitud de sus

Anagramas, Volumen 9, № 17, pp. 45-56 - ISSN 1692-2522 - Julio-diciembre de 2010. 166 p. Medellín, Colombia 
hijos (27,3\%). Los datos revelan que el porcentaje de mujeres que instan a sus padres a comprarles un celular, $(34,9 \%)$ es superior al número de hombres, $(28,4 \%)$. Por el contrario, cuando son los progenitores quienes toman la iniciativa no hay relevante diferencia en cuanto al género. El regalo por parte de algún familiar también es una vía importante de acceso, aunque muy por detrás de los padres $(13,1 \%)$.

Derivado de lo anterior no resulta extraño que el 58,3\% de los adolescentes, (56,2\% de hombres, frente al 60,1\% de mujeres) declare que nunca discute con sus padres con motivo del celular. Esto significa que, en general, este dispositivo no es una fuente de conflicto familiar. No obstante, entre quienes sí reconocen discusiones, el dinero que gastan y el tiempo que dedican a su uso son los principales motivos de discusión. Un 11,8\% de las mujeres admitió discutir por el dinero gastado ante el 9,5\% de los hombres. El 15,4\% de ellas admitió tener disputas con sus padres por el tiempo dedicado a usar el celular, frente al 13,7\% de los hombres

Casi la mitad de los adolescentes colombianos $(47,8 \%)$ aseguró desconocer el gasto que realiza con su teléfono celular. Por otra parte, el 44,7\% reconoce en sus padres a los financiadores del servicio, siendo mayor el apoyo económico para los mujeres (50\%) frente al 37,9\% de los hombres. Sólo el 21 ,4\% de los adolescentes colombianos lo pagan con sus propios recursos, $24,7 \%$ entre los varones y 19\% entre las féminas.

\section{Uso del dispositivo}

En general, los adolescentes colombianos entienden el celular más como un aparato para comunicarse, que como un dispositivo con funciones lúdicas. Por ello, las funciones más utilizadas son los servicios de voz y de mensajería de texto SMS. En cuanto a género, el porcentaje de mujeres que utilizan el móvil para llamar y recibir llamadas es casi 9 puntos porcentuales mayor al de los hombres. En el uso del móvil para enviar SMS, la diferencia casi alcanza los 12 puntos porcentuales.

Aludiremos también a la función lúdica que los menores pueden otorgar a este aparato. A muchos adolescentes el celular les sirve para jugar, escuchar música, hacer fotos, ver fotos y vídeos o grabar vídeos. De hecho, el uso del celular 'para jugar' es una de las actividades más habituales entre los adolescentes, sólo superada por las acciones comunicativas mencionadas en el párrafo anterior. Un 48,5\% de los encuestados afirmó que el celular le servía para ello. Existe, por tanto, ese uso del celular como modo de divertirse y entretenerse, aunque en un porcentaje inferior a su función comunicativa original. En la mayoría de los usos incluidos en este segundo grupo, el porcentaje de mujeres supera al de hombres.

Por último, otra de las aplicaciones que resultan destacables y que muchos adolescentes declaran llevar a cabo con su celular son el reloj y la agenda. La función de organizador del tiempo juvenil es, según los resultados obtenidos, la tercera función más habitual que otorgan a su teléfono. Estos usos del celular son también más frecuentes entre las mujeres que entre los hombres, como queda reflejado en el gráfico 3 (Gráfico 3. Usos del celular 10 a 18 años).

Gráfico 3. Usos del celular

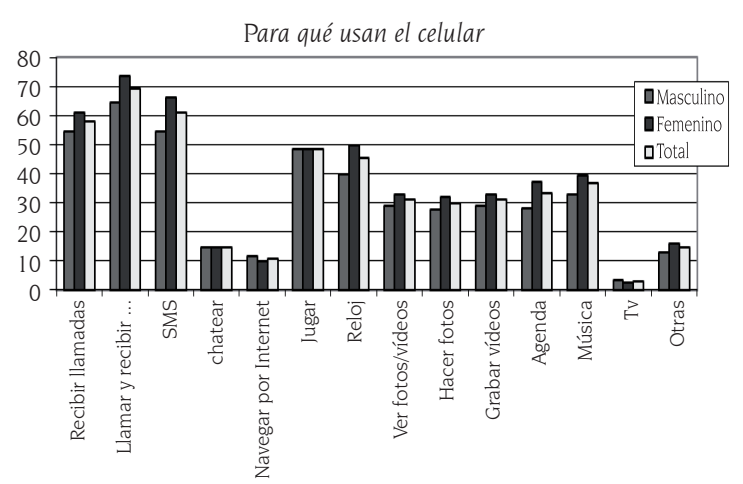

Fuente: Encuestas Generaciones Interactivas en Iberoamérica. Respuesta a la pregunta No 33 "El celular te sirve principalmente para...". $\mathrm{N}=3.292$ escolares de 10 a 18 años.

\section{Interlocutores}

La mayoría de los adolescentes colombianos tiene como principal interlocutor a su madre, 60,2\%, porcentaje superior incluso a los que consideran a sus amigos con este término, 53,6\%. Estos datos revelan un incremento muy significativo en la comunicación que mantienen los menores colombianos con sus madres e iguales en este tramo de 
edad (entre los 10 y los 18 años), sobre todo, si se comparan estos datos con los obtenidos sobre esta misma cuestión en la muestra de los niños menores de 10 años. El porcentaje no sorprende en lo que se refiere a iguales, puesto que es en la adolescencia cuando los amigos cobran un mayor protagonismo llegando a ser el foco principal de relación, donde uno se conoce, reconoce y va definiendo su propia identidad.

Sin embargo, la comunicación con los progenitores también aumenta en este tramo de edad, siendo muy significativa en el caso de la madre, donde las conversaciones con ella se amplían con respecto al tramo de 6 a 9 años en más de 20 puntos porcentuales.

Por último, es relevante destacar que el porcentaje de mujeres que afirma hablar con los diversos interlocutores propuestos supera en todos los casos al de los hombres, con una única excepción: la comunicación con la pareja, donde los porcentajes coinciden, 27\% en ambos casos. La divergencia porcentual entre féminas y varones es muy abultada, sobre todo, en lo referente a las conversaciones que los adolescentes mantienen con sus amigos, (61,2\% de mujeres, frente al 44\% de hombres), y con sus madres, $(63,8 \%$ frente, al 55,6\%).

\section{Disponibilidad}

Podemos afirmar que existe cierto consenso entre los adolescentes colombianos sobre los momentos en que conviene apagar el celular. El 39,6\% asegura desconectarlo en clase; el 25,4\% cuando está en el cine; y sólo el 12,7\% cuando está en la cama. Sorprende también que sólo un 19,5\% afirme apagarlo durante los ratos de estudio personal. En cuanto al género, no se aprecian diferencias significativas alrededor de esta decisión. Los datos evidencian también que, aunque las diferencias porcentuales por género no superan en ningún caso los 5 puntos, los hombres sobresalen frente a las mujeres en todos los supuestos en los que consideran necesario apagar su celular.

También es significativo que un 30,3\% de ellos asegure que siempre está dispuesto a utilizarlo, independientemente del momento que sea. El porcentaje de mujeres dispuestas a emplearlo siempre, $(35,6 \%)$ supera considerablemente al de los hombres que se adhieren a dicha afirmación, $(23,5 \%)$. La disponibilidad aumenta todavía más cuando se trata de comunicar con sus amigos. De hecho, ante la afirmación de "siempre tengo el celular encendido para comunicar con mis amigos", un 44,3\% de los adolescentes colombianos contestó afirmativamente, siendo una vez más considerablemente superior el número de mujeres $(50,1 \%)$, frente al de hombres, $(36,7 \%)$. Todos estos datos evidencian la utilidad social de comunicación con iguales que los adolescentes colombianos otorgan a sus celulares.

Por último, a pesar de la relevante penetración del celular entre los adolescentes colombianos, y del gran uso social que hacen del mismo, lo cierto es que la gran mayoría $(62,5 \%)$ es consciente de que su vida no cambiaría para bien o para mal si prescindieran del aparato durante dos semanas.

\section{Videojuegos}

El 72,9\% de los adolescentes colombianos asegura que juega habitualmente con videojuegos o juegos de computador. Estamos, por tanto, ante una pantalla que goza de gran penetración entre este grupo de la población, aunque esa realidad se hace todavía más evidente entre los hombres con un $83,5 \%$ frente al $64,7 \%$ de las mujeres.

El computador $(52,5 \%)$, el celular $(52,2 \%)$ y la videoconsola $(38,1 \%)$ son, por este orden, las plataformas preferidas por los jugadores. Si analizamos esta realidad introduciendo la variable género, vemos que la dinámica descrita varía en los soportes que los adolescentes eligen en primer y tercer lugar, como se aprecia en la tabla 4. Las mujeres emplean como plataforma prioritaria el computador, seguida del celular y de Internet. En el caso de los hombres, la videoconsola pasa a ser la primera plataforma más frecuente, seguida del celular y en tercer lugar de la computadora.

Por último, se podría destacar la penetración de las videoconsolas en Colombia, que se encuentra casi en la media latinoamericana situada en un 39\%. En el caso de los adolescentes varones la media es rebasada en casi 10 puntos porcentuales. 
Tabla 2. Plataformas de uso de videojuegos

\begin{tabular}{|c|c|c|c|c|c|c|c|}
\hline & Videoconsola & Computadora & $\begin{array}{c}\text { Videoconsola } \\
\text { portátil }\end{array}$ & Internet & Celular & Mp3 & Otros \\
\hline Masculino & $48,1 \%$ & $47,5 \%$ & $15,3 \%$ & $31,2 \%$ & $48 \%$ & $14,7 \%$ & $19,5 \%$ \\
\hline Femenino & $27,9 \%$ & $58 \%$ & $14,8 \%$ & $34,8 \%$ & $57 \%$ & $23,2 \%$ & $22,1 \%$ \\
\hline Total & $38,1 \%$ & $52,5 \%$ & $15,1 \%$ & $33,0 \%$ & $52,2 \%$ & $18,9 \%$ & $20,9 \%$ \\
\hline
\end{tabular}

Fuente: Encuestas Generaciones Interactivas en Iberoamérica. Respuesta a la pregunta № 42 «¿Con qué?». N= 3.292 escolares de 10 a 18 años

\section{Tiempo}

Los datos que aporta la investigación permiten establecer diferencias en el tiempo que los adolescentes colombianos destinan a jugar con los videojuegos entre semana y los fines de semana. El porcentaje que invierte menos de una hora diaria a esta actividad se reduce durante los fines de semana en casi 8 puntos porcentuales. En el caso de los médium users, el porcentaje es casi idéntico durante los días laborales y festivos: 17,9\% entre semana; $18 \%$ los fines de semana. Sin embargo, el porcentaje de heavy users aumenta en Colombia durante los festivos, pasando de un $10,8 \%$, a un $14,9 \%$.

El tiempo diario dedicado a los videojuegos también varía considerablemente en función del género. La mayor parte de los hombres que juegan de lunes a viernes lo hace entre una y dos horas $(21,7 \%)$, seguidos de los que juegan menos de una hora (20\%). Un 13,5\% de ellos admiten jugar más de dos horas. Por el contrario, entre semana, la mayoría de las mujeres afirman jugar menos de una hora $(30,6 \%)$, siendo el $14 \%$ de ellas la que admiten destinar entre una y dos horas a esta actividad. Sólo un 8\% de las féminas juegan más de dos horas.

Durante los fines de semana el porcentaje de hombres que juegan a videojuegos se reducen en todas las categorías analizadas a excepción de los heavy user. Si entre semana un 13,5\% de los adolescentes varones se incluían en esta categoría, el fin de semana el porcentaje aumenta hasta el 19,5\%.

En el caso de las mujeres, son las medium user y las heavy user las que aumentan algo su consumo de los videojuegos los fines de semana: del 14\% al $15,1 \%$ entre las primeras; y del $8 \%$ al $10,3 \%$ en la segunda categoría mencionada.
Casi la mitad de los adolescentes encuestados $(49,1 \%)$ admiten que nunca discuten con sus padres por cuestiones relacionadas con los videojuegos, siendo significativa la diferencia entre mujeres y hombres: un 59,7\% de ellas admitió no tener disputas nunca, porcentaje que disminuye hasta el 39,1\% en el caso de los hombres. El problema del tiempo se constituye en la principal fuente de discusión entre padres e hijos. El 36,5\% de los varones y el 19,9\% de las féminas admite discutir por este motivo. Merece la pena destacar también que en todas las problemáticas que pueden generar discusiones que se han analizado, el porcentaje de hombres que discuten con sus padres supera siempre al de las mujeres.

El hecho de que más un 33,2\% de los adolescentes encuestados $(35,6 \%$ hombres, $30,8 \%$ mujeres) afirme que los videojuegos les quitan tiempo de estudio ayuda a comprender que el espacio dedicado a los videojuegos sea el foco prioritario de discusión entre padre e hijos. Y es que, para muchos progenitores, como ya hemos constatado, el tiempo dedicado es el principal escollo, por encima de otras fuentes de conflicto como pueden ser el contenido de los videojuegos o el momento escogido para jugar, donde el porcentaje de menores que admiten discutir por ello se reduce significativamente hasta porcentajes siempre inferiores al 17\%.

Conforme los niños van creciendo, la importancia individual del juego aumenta sin que la experiencia social del mismo entre iguales decrezca (sobre todo entre amigos y hermanos). A pesar de que la opción de juego en solitario sigue siendo la más habitual, en ambos sexos, la compañía de los amigos también se incrementa considerablemente en este tramo de edad con respecto a los porcentajes obtenidos entre menores de 9 años. 
Se produce además un descenso porcentual muy considerable si analizamos la experiencia social del juego de los adolescentes con sus progenitores y los comparamos con los datos obtenidos en la muestra de niños colombianos de 6 a 9 años.

También merece la pena reseñar que los hombres $(52,7 \%)$ son algo más proclives a jugar en soledad que las mujeres $(51,4 \%)$. Por el contrario, el número de mujeres que juegan con otras personas supera en todos los casos al número de hombres que lo hacen acompañados, siendo la excepción a esta afirmación el caso del juego compartido con amigos. En este caso, tal y como se muestra en el siguiente gráfico, el porcentaje de varones supera al de féminas (Gráfico 4. Compañía en el uso de videojuegos).

Gráfico 4. Compañía en el uso de videojuegos Con quién suelen jugar a videojuegos

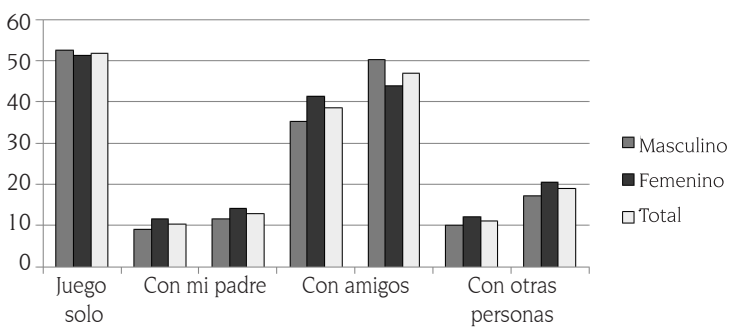

Fuente: Encuestas Generaciones Interactivas en Iberoamérica. Respuesta a la pregunta $\mathrm{N}^{\circ} 48$ «¿Con quién sueles jugar?». $\mathrm{N}=3.292$ escolares de 10 a 18 años.

Consideración aparte merecen los juegos en red, que gozan de bastante popularidad entre este público, ya que más del $40 \%$ de los adolescentes colombianos asegura que habitualmente los utiliza con su grupo de amigos. El sesgo por género vuelve a ser visible en esta pregunta: el 43,3\% de hombres, frente al $37,6 \%$ de mujeres reconoce jugar en red con sus amigos. La preferencia por esta modalidad de juegos puede estar relacionada con el componente social que lleva aparejada. De hecho, un 22,4\% de los hombres y el 19,1\% de las mujeres afirma que jugar en red permite hacer amigos, al mismo tiempo que es más divertido porque hay más gente jugando: 32,4\% hombres y $23,8 \%$ mujeres. Esto significa que a una buena parte de los adolescentes le resulta atractivo el hecho de compartir la experiencia con otros y no jugar en soledad, aunque como ya hemos dicho, algo más de la mitad de la muestra opta también por jugar sin compañía.

\section{Conclusiones}

Todos los datos aportados en este estudio confirman la penetración que las nuevas tecnologías de información y comunicación han alcanzado entre el público adolescente colombiano, tanto en hábitos de consumo de servicios relacionados con Internet, telefonía celular y videojuegos, como en las preferencias manifiestas por cierto tipo de contenidos y aplicaciones ofrecidos por estas plataformas.

A diferencia de lo demostrado por estudios aplicados en países desarrollados como Estados Unidos, la penetración de las nuevas tecnologías de información y comunicación no ha sustituido, entre los adolescentes colombianos, a la comunicación personalizada tanto entre sus semejantes como con sus progenitores. No obstante, es de resaltar que estos últimos manifiestan mayor preocupación por el tiempo que sus hijos emplean en el consumo de estas tecnologías, que por el tipo de contenidos a los que tienen acceso y por los cuales se sienten atraídos.

Se destaca igualmente el contraste entre la falta de una política educativa para la orientación y capacitación para el acceso y el manejo de estas tecnologías, y la recursividad manifiesta y evidenciada por los adolescentes para obtener instrucción básica que les impida quedar al margen de esta recursividad tecnológica.

No obstante, preocupa que el mayor destino que los adolescentes colombianos le dan a estas tecnologías esté enfocado más hacia el ocio, el entretenimiento y la recreación, mientras se desperdicia este recurso con fines de formación personal y de educación formal, en un grupo de edad privilegiado para estos propósitos.

Finalmente, la dinámica cambiante de la relación establecida por los diferentes públicos con las nuevas tecnologías de información y comunicación obliga a practicar estudios en forma periódica, con el fin de poder interpretar de 
manera adecuada los cambios y los fenómenos presentados en la nueva comunicación del siglo XXI.

\section{Referencias bibliográficas}

Arango Forero, G., \& González Bernal, M. (2009). Televidencias juveniles en Colombia: fragmentación generada por un consumo multicanal. [Investigación]. Palabra Clave, 12(2), pp. 215-234.

Arango Forero, G. (2008). Colombia. En: X. Bringué \& C. Sábada (Eds.), La Generación interactiva en Iberoamérica: niños y adolescentes ante las cámaras. (pp. 262). Barcelona: Ariel.

Bringué, X. (2008). Niños y jóvenes en un nuevo escenario de comunicación. En: C. Etayo, E. Moreno, J. E. Guerrero, C. Sánchez Blanco \& M. Navarro (Eds.), Los jóvenes y el nuevo escenario de la comunicación. Actas del XXI Congreso Internacional de la Comunicación. Pamplona: Eunsa.

Bringué, X., E Sábada, C. (2008). La generación interactiva en Iberoamérica. Pamplona: Ariel.

Casetti, F. (1999). Análisis de la televisión. Instrumentos, métodos y prácticas de investigación. Barcelona: Paidós. 384 p. Análisis de la televisión. Instrumentos y prácticas de investigación.

Ley 1341, por la cual se definen principios y conceptos sobre la sociedad de la información y la organización de las Tecnologías de la Información y las Comunicaciones -TIC-, se crea la Agencia Nacional de Espectro y se dictan otras disposiciones (2009).

Fosk, A. (2010). Situación de Internet en Latinoamérica. Disponible en: http://www.slideshare.net/ francoalfero/estado-de-internet-en-latinoamericacomscore-2010.

Livingstone, S. (2005). Audiences and Publics, when cultural engagement matters for the Public Sphere (Vol. 2). Bristol, U.K.: Intellect Books.

Ontiveros Baeza, E., Martín Enríquez, A., Fernández de Lis, S., Rodríguez Téubal, I., E López Sabater, V. (2009). Telefonía móvil y desarrollo financiero en América Latina. Barcelona: Ariel.

Scolari, C. (2007). La televisión, ese fenómeno "masivo" que conocimos, está condenada a desaparecer. Entrevista a Eliseo Verón, realizada por Carlos Scolari y Paolo Bertetti. (En línea). Recuperado el domingo 21 de febrero. Disponible en http://www.modernclicks.net/weblog/mediamerica-entrevista_veron. pdf.

Tolsá, J. (2008). La generación interactiva como objeto de estudio. En: X. Bringué \& C. Sábada (Eds.), La generación interactiva en iberoamérica. Niños y adolescentes ante las pantallas (pp. 205-223). Barcelona: Ariel.

Turow, J. (2010). Media Today: an introduction to mass communication (Vol. 3). New York: Routledge.

Vergara, Y. C. (2009). Telemedellín 2.0: Una mirada a la televisión en Internet. (En línea) recuperado el jueves 12 de noviembre. Disponible en http://mediosdigitalesmedellin.blogspot.com/. 